\title{
Gastric cancer-derived mesenchymal stromal cells trigger M2 macrophage polarization that promotes metastasis and EMT in gastric cancer
}

\author{
Wei $\mathrm{Li}^{1,2}$, Xu Zhang ${ }^{3}$, Fenglei Wu${ }^{4}$, Ying Zhou' ${ }^{1}$ Zengtao Bao ${ }^{5}$, Haining $\mathrm{Li}^{1,6}$, Ping Zheng ${ }^{1}$ and Shaolin Zhao
}

\begin{abstract}
Resident macrophages in the tumor microenvironment exert a dual role in tumor progression. So far, the mechanism of intratumoral macrophage generation is still largely unknown. In the present study, the importance of macrophages in the pro-tumor role of gastric cancer-derived mesenchymal stromal cells (GC-MSCs) was observed in a mouse xenograft model with macrophage depletion. In gastric cancer tissues, high expression levels of Ym-1, Fizz-1, arginase1, and CCR-2, as well as a low expression level of iNOS, were verified, and co-localization of GC-MSCs and tumorassociated macrophages (TAMs) was observed by dual immunofluorescence histochemistry. TAMs isolated from gastric cancer tissues predominantly displayed an M2 phenotype. In a co-culture system, the contribution of GC-MSCS to $\mathrm{M} 2$ polarization of macrophages was confirmed by the M2-related protein expression, M2-like immunophenotype and cytokine profile of GC-MSC-primed macrophages in vitro. Blockade of IL-6/L-8 by neutralizing antibodies significantly attenuated the promoting effect of GC-MSCs on M2-like macrophage polarization via the JAK2/ STAT3 signaling pathway. In addition, GC-MSC-primed macrophages promoted the migration and invasion of gastric cancer cells, and the process of EMT in gastric cancer cells was significantly enhanced by GC-MSC-primed macrophage treatment. Our study showed that tumor-promoting GC-MSCs contribute to M2 macrophage polarization within the gastric cancer niche through considerable secretion of IL-6 and IL-8. These GC-MSC-primed macrophages can subsequently prompt gastric cancer metastasis via EMT promotion in gastric cancer cells.
\end{abstract}

\section{Introduction}

Gastric cancer is the second most prevalent malignant tumors worldwide ${ }^{1}$. Although advancement in treatment has resulted in a decrease in mortality, the 5-year survival rate of patients after surgical resection remains low $^{2}$. Among the causes, a high rate of metastasis is a major obstacle in improving long-term survival after curative resection $^{3}$. Therefore, elucidation of a unique pathological mechanism for gastric cancer progression is urgently

\footnotetext{
Correspondence: Wei Li (medical112@126.com) or

Shaolin Zhao (zhaoshaolin1@163.com)

${ }^{1}$ Center of Research Laboratory, The First People's Hospital of Lianyungang, Lianyungang 222001, China

${ }^{2}$ Department of Pathology, Xuzhou Medical University, Xuzhou 221004, China

Full list of author information is available at the end of the article.

These authors contributed equally: Wei Li, Xu Zhang, Fenglei Wu

Edited by H.-U. Simon
}

needed to search for effective therapeutic targets for gastric cancer. Accumulating evidence suggests that macrophages are critical for the occurrence and development of various tumors ${ }^{4,5}$. A high rate of macrophage infiltration is associated with poor prognosis of gastric cancer and the markers of macrophages can be used as prognostic indexes.

In diverse tumor stroma, tumor-associated macrophages (TAMs) have high plasticity and predominantly present as an M2 phenotype, which is associated with cancer metastasis and worse prognosis in patients ${ }^{7,8}$. However, the underlying mechanism for M2-like TAM generation within the tumor microenvironment is largely unknown. The effects of cancer cells on the M2 polarization of macrophages have become a research focus. Factors secreted by cervical cancer cells have been

\section{(c) The Author(s) 2019}

(c) (i) Open Access This article is licensed under a Creative Commons Attribution 4.0 International License, which permits use, sharing, adaptation, distribution and reproduction in any medium or format, as long as you give appropriate credit to the original author(s) and the source, provide a link to the Creative Commons license, and indicate if changes were made. The images or other third party material in this article are included in the article's Creative Commons license, unless indicated otherwise in a credit line to the material. If material is not included in the article's Creative Commons license and your intended use is not permitted by statutory regulation or exceeds the permitted use, you will need to obtain permission directly from the copyright holder. To view a copy of this license, visit http://creativecommons.org/licenses/by/4.0/. 
demonstrated to induce a stable M2 phenotypic profile in THP-1-derived macrophages in vitro ${ }^{9}$. Breast cancerderived hyaluronan also stimulates the formation of M2like TAMs in a mouse model ${ }^{10}$. Peritoneal TAMs are also polarized to an M2 phenotype by gastric cancer cells ${ }^{11}$. Nevertheless, the contribution of tumor stromal cells to the generation of M2-like TAMs is still obscure and could be critical for identifying better potential therapeutic targets in gastric cancer.

Tumor stromal cells have been reported to exert an important role in the initiation and progression of various tumors $^{12,13}$. As one of the key components in tumor stroma, mesenchymal stromal cells (MSCs) have attracted much attention and their unique effect on tumorigenesis and progression has been noted ${ }^{14,15}$. Within the tumor microenvironment, MSCs exert their immunosuppressive potential via interacting with a variety of immune cells, such as lymphocytes, dendritic cells and macrophages ${ }^{16,17}$. One of our previous studies indicated that human umbilical cord-derived stromal cells can notably promote M2 polarization of macrophages at sites of renal ischemia-reperfusion injury ${ }^{18}$. Following that, in another study, we isolated cancer-associated MSCs from gastric cancer tissues, named as gastric cancer-derived MSCs (GC-MSCs), and found their ability to promote the proliferation, migration and angiogenesis of gastric cancer cells; they are more potent than non-cancerous tissuederived $\mathrm{MSCs}^{19}$. Thereafter, we investigated the regulatory effect of GC-MSCs on M2-like TAM generation in gastric cancer and, subsequently, the role of GC-MSCprimed macrophages in prompting tumor progression.

In this study, we reported for the first time that the polarization and generation of pro-tumor M2-like macrophages was strikingly triggered by GC-MSCs in gastric cancer through activation of the JAK2/STAT3 signaling pathway via high secretion of IL-6/IL-8. Moreover, GCMSC-primed macrophages remarkably promoted gastric cancer metastasis by enhancing the process of EMT in gastric cancer cells.

\section{Results}

Macrophages are essential for the tumor-promoting effect of GC-MSCs in vivo

GC-MSCs were characterized by their pluripotent differentiation potential and immunophenotype. After 7-14 days of primary culture, the cells emerged with fibroblast-like morphology, with both osteoblastic and adipogenic differentiation potential, and the cells were positive for CD29, CD44, CD90, and CD105 but negative for CD14, CD34, and CD45 (Supplementary Fig. 1). To assess the contribution of macrophages to the pro-tumor effect of GC-MSCs, we performed tumor xenograft experiments in BALB/c nude (nu/nu) mice with concomitant depletion of monocytes/macrophages by lipo-
$\mathrm{Cl}_{2} \mathrm{MBP}$, which is a macrophage 'suicide' agent developed by van Rooijen et al. ${ }^{20}$. As shown in Fig. 1a, monocytes/ macrophages were depleted in each cohort of tumorbearing mice by lipo- $\mathrm{Cl}_{2} \mathrm{MBP}$ application via intravenous injection at different time points during the experimentation period. After subcutaneous co-injection with GC-MSCs at a 5:1 ratio for 14 days, BGC-823-implanted mice displayed significant increases in either tumor weight or volume compared with the control cohort of mice (Fig. 1b-d). However, macrophage depletion strikingly abrogated the tumor-promoting effect of GC-MSCs (Fig. 1b-d). In agreement with this, a more severe histopathology was observed by H\&E staining in the cohort of mice with BGC-823 and GC-MSC co-injection than in the control cohort, and the severity of malignant tumor significantly decreased upon macrophage depletion (Fig. 1e). The efficiency of myeloid cell depletion by lipo- $\mathrm{Cl}_{2} \mathrm{MBP}$ was confirmed by analyzing tumor tissues for $\mathrm{F} 4 / 80^{+}$cell infiltration (Fig. 1e). The results of Ki67 and CD31 staining indicated increased intratumor proliferation and angiogenesis, respectively, in the cohort of mice with co-injection compared with the control cohort, which was also abrogated by macrophage depletion (Fig. 1e). In another mouse xenograft model with MKN28, similar results were also observed (Supplementary Fig. 2). Thus, our results indicate a key role of macrophages in the potent pro-tumor effect of GC-MSCs. The properties of TAMs and the potential contribution of GC-MSCs to TAM generation within gastric cancer drew our attention.

\section{TAMs in gastric cancer with GC-MSC co-existence predominantly present with the M2 subtype}

To study the potential correlation between GC-MSCs and macrophages, we expanded our observation to include tumor tissues from 10 randomly selected clinical gastric cancer patients. Compared with the adjacent noncancerous tissues, significantly higher levels of Ym-1, Fizz-1, arginase-1 and CCR-2, the M2 macrophages' markers, were observed in gastric cancer tissues with GCMSCs infiltration (Fig. 2a, b). We also collected tumor tissues from gastric cancer cell-implanted mice at 14 days. Compared with the control cohort, the expression level of iNOS mRNA was significantly lower in the experimental cohort with GC-MSC co-injection, whereas Ym-1, Fizz-1 and arginase-1 were all highly expressed in the coinjection cohort (Supplementary Fig. 3). In addition, the confocal immunofluorescence images displayed an obvious co-localization of GC-MSCs and M2-type macrophages within tumor tissues collected from three representative gastric cancer patients (Fig. 2c).

Thereafter, the infiltrated macrophages were further sorted by magnetic cell sorting (MACS) in either the cancer tissues or the corresponding non-cancerous tissues. The flow cytometric assay displayed a higher ratio of 
A Subcutaneous injection of cells

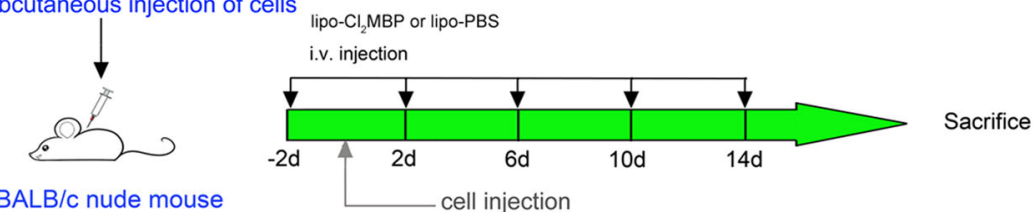

B

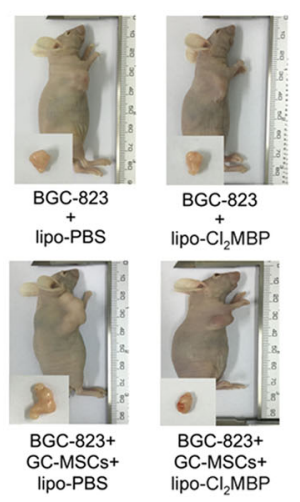

C

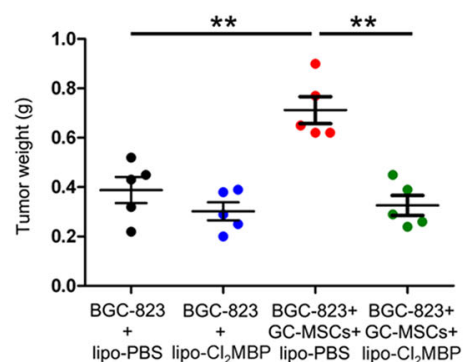

$\stackrel{+}{+} \stackrel{+}{+}$ GC-MSCs+ GC-MSCs+
lipo-PBS lipo-Cl
$\mathrm{Cl}_{2} \mathrm{MBP}$ lipo-PBS lipo-Cl MBP

$\mathbf{E}$
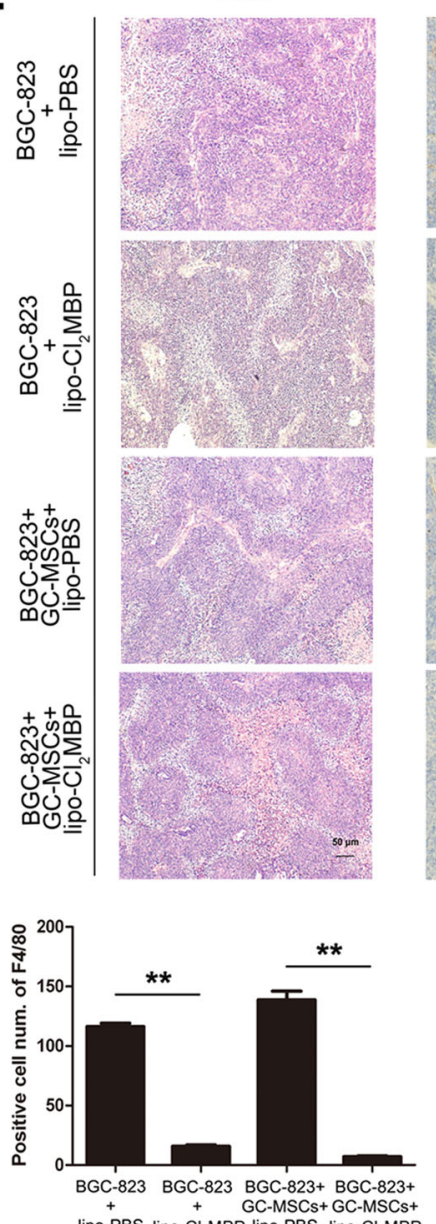

$\stackrel{+}{+} \underset{\text { lipo-PBS }}{+} \stackrel{+}{\text { GC-MSCs+ GC-MSCs+ }}+$
$\mathrm{F} 4 / 80$
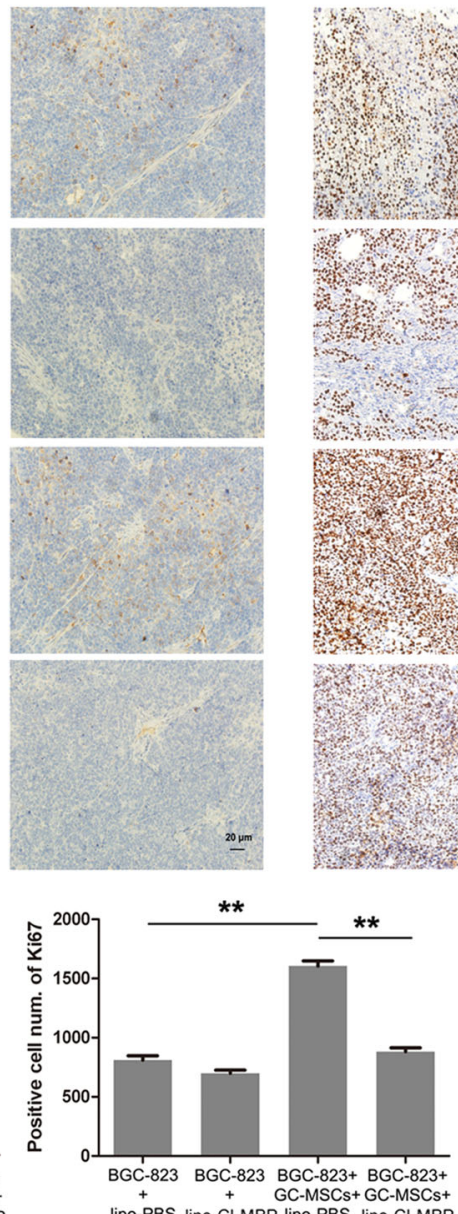

D

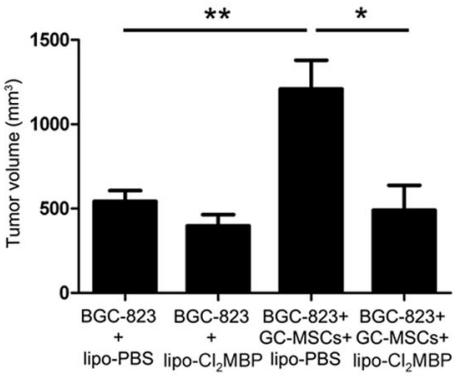

Ki67

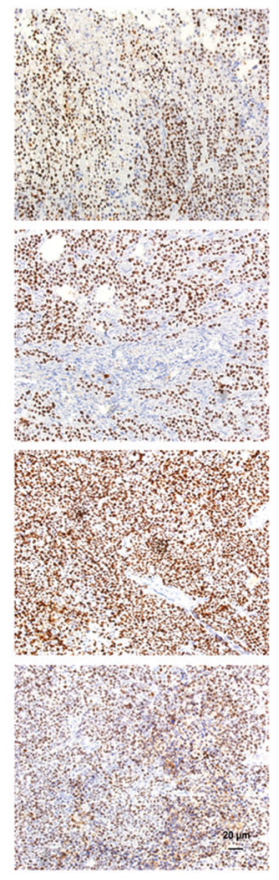

CD31
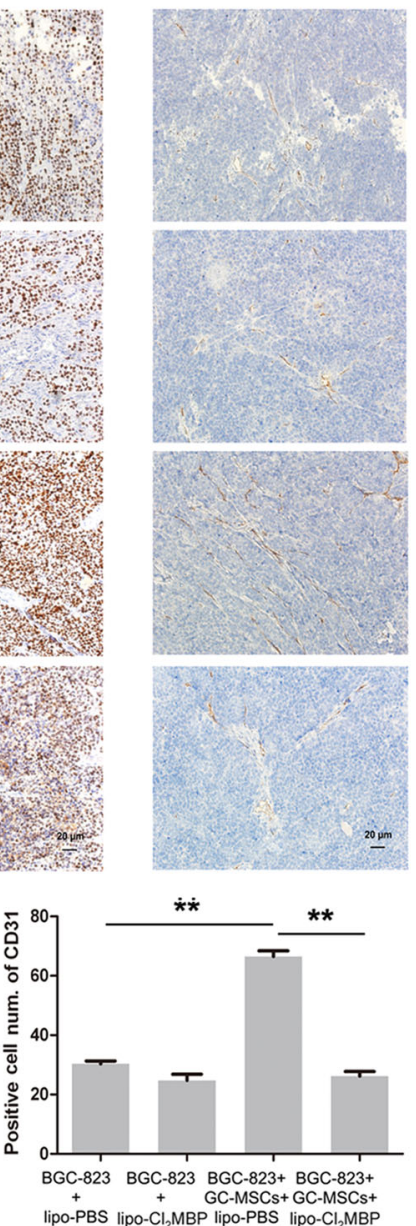

Fig. 1 (See legend on next page.) 
(see figure on previous page)

Fig. 1 Macrophage-depletion dampens tumor growth facilitated by GC-MSCs in a mouse xenograft model. a Experimental design of macrophage-depletion by clodronate liposome application in gastric cancer-bearing BALB/c nude mice. $\mathbf{b}$ Representative gross morphology of tumor at 14 days after BGC-823 transplantation with distinct treatments. c Tumor weight and $\mathbf{d}$ tumor volume evaluated in each group of mice at 14 days after BGC-823 transplantation with distinct treatments. $n=5$ in each group. e Representative histopathology images of tumors collected from mice co-injected by BGC-823 and GC-MSCS, with or without macrophage-depletion. H\&E staining: scale bar, 50 um; Immunohistochemistry for F4/80: scale bar, $20 \mu \mathrm{m}$; for Ki67: scale bar, $20 \mu \mathrm{m}$; for CD31: scale bar, $20 \mu \mathrm{m} .{ }^{*} P<0.05 ;{ }^{*} P<0.01$.

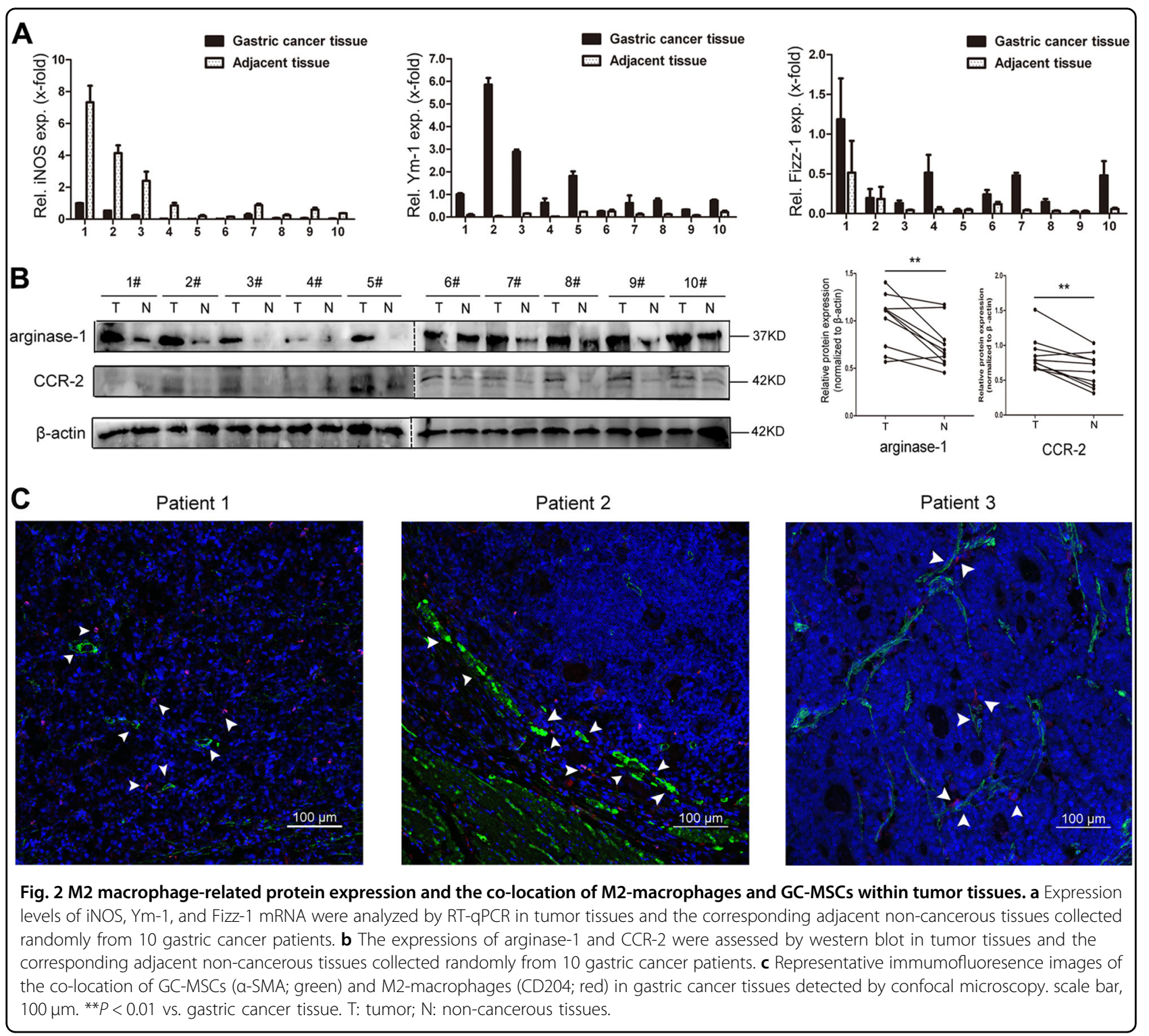

CD204 ${ }^{+}$cells in macrophages derived from the human gastric cancer tissues than in macrophages derived from the corresponding non-cancerous tissues (Fig. 3a). RTqPCR showed significantly higher levels of CD163, CD204, CD206, IL-10, and CCL-22 mRNA, which indicates an M2-like immunophenotype, in macrophages selected from the gastric cancer tissues than in those from the adjacent non-cancerous tissues, whereas the expression level of the M1-subtype marker IL-23 was significantly lower in gastric cancer tissue-derived macrophages (Fig. 3b). Next, we wondered whether the characterized phenotype of peripheral monocytes is 


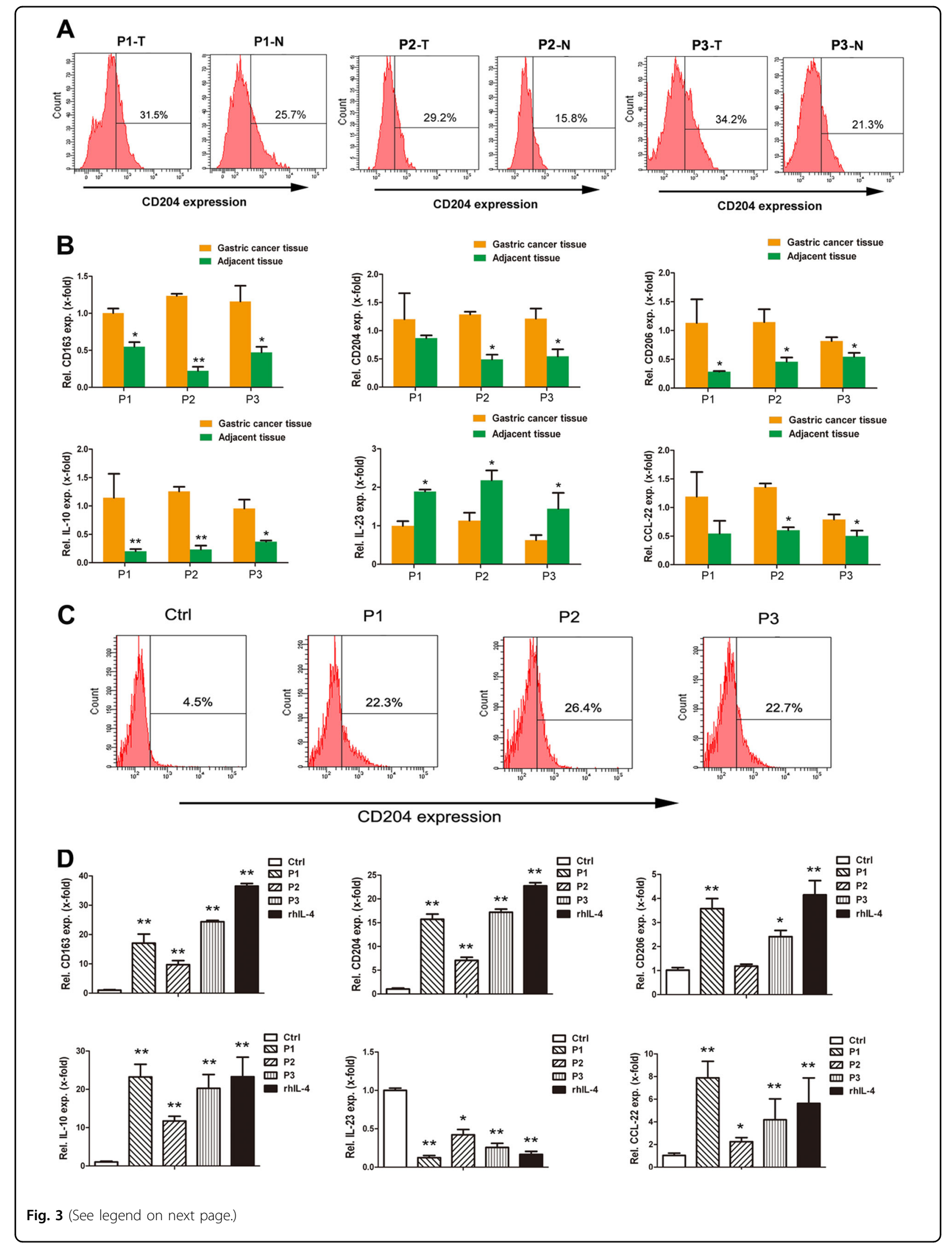


(see figure on previous page)

Fig. 3 Macrophages picked up from tumor tissue or peripheral blood of gastric cancer patients present as a M2-like phenotype

prominently. a The proportion of $\mathrm{CD} 204^{+}$cells was detected by flow cytometry in macrophages harvested by MACS from tumor tissues and the corresponding adjacent non-cancerous tissues of three representative gastric cancer patients. b Expression levels of CD163, CD204, CD206, IL-10, CCL-22, and IL-23 mRNA were detected by RT-qPCR in macrophages of tumor tissue and the corresponding adjacent non-cancerous tissue origin. c The proportion of $\mathrm{CD}_{2} \mathrm{O4}^{+}$cells was detected by flow cytometry in macrophages of peripheral origin harvested by MACS from three representative gastric cancer patients and the healthy control. d Expression levels of CD163, CD204, CD206, IL-10, CCL-22, and IL-23 mRNA were detected by RTqPCR in macrophages of peripheral origin. ${ }^{*} P<0.05 ;{ }^{*} P<0.01$ vs. Ctrl. T: tumor; N: non-cancerous tissues; Ctrl: control; $P$ : patient.

different in gastric cancer patients and healthy controls, and the ratio of $\mathrm{CD} 204^{+}$cells was shown to be strikingly higher in macrophages collected from the peripheral blood of gastric cancer patients (Fig. 3c). RT-qPCR results also showed higher levels of CD163, CD204, CD206, IL10 , and CCL-22 mRNA in peripheral blood-derived macrophages selected from gastric cancer patients than from healthy controls (Fig. 3d). These observations suggest that GC-MSCs may play a role in M2 macrophage polarization in gastric cancer progression.

\section{GC-MSCs promote the polarization of macrophages into an M2-like subtype in vitro}

To confirm whether M2 macrophage polarization is triggered by GC-MSCs, a co-culture system including GC-MSCs and human peripheral blood monocytederived macrophages was further exploited in our study. RT-qPCR results demonstrated higher expression levels of representative M2 macrophage markers in macrophages after co-culture with GC-MSCs for 3 days (Fig. 4a). Flow cytometric assays showed that GC-MSC treatment significantly increased the ratio of $\mathrm{CD} 204^{+} / \mathrm{CD} 163^{+}$cells in macrophages at 3 days (Fig. 4b). In addition, by Luminex assay analysis, the concentrations of proinflammatory cytokines TNF- $\alpha$, IP-10, RANTES, and MIP- $1 \alpha$ significantly decreased in the supernatant of macrophages co-cultured with GC-MSCs, whereas the secretions of IL-6, IL-10, VEGF, and MCP-1, which are in favor of tumor progression, increased notably (Fig. 4c). Therefore, we demonstrated that GC-MSCs, one of the key pro-tumor stromal cell types, may play a potent role in inducing the M2 macrophage polarization within the gastric cancer microenvironment.

\section{GC-MSC-primed macrophages promote gastric cancer metastasis more potently than GC-MSCs do}

One of our previous reports conveyed that GC-MSCs strikingly promote the proliferation and migration of gastric cancer cells, and this promotion is more potent than that in GCN-MSCs or bone marrow-derived MSCs $(\mathrm{BM}-\mathrm{MSCs})^{19}$. In this study, we observed that macrophages play an essential role in the tumor-promoting effect of GC-MSCs, and that GC-MSCs could in turn potently prompt the conversion of macrophages into the tumor-supporting M2 subtype. Thus, we investigated the potential roles of GC-MSC-primed macrophages in gastric cancer process and progression. As shown in Fig. $4 \mathrm{~d}$, CM from GC-MSC-primed macrophages significantly enhanced the proliferation of gastric cancer MKN-28 cells at either 3 days or 5 days of co-culture. However, the tumor-promoting ability of GC-MSC-primed macrophage$\mathrm{CM}$ was not more potent than that of GC-MSC-CM (Fig. 4d). Similar results regarding the proliferative ability of MKN-28 cells with diverse treatments were obtained when examining PCNA expression by western blot (Fig. 4e). Transwell migration assays indicated that the number of migrated MKN-28 cells strikingly increased with GC-MSCprimed macrophage-CM, which was even more potent than with GC-MSC-CM (Fig. 4f). MKN-28 cells treated with GC-MSC-primed macrophage-CM also showed the highest cell invasion ability (Fig. 4g). Thus, GC-MSCs can regulate the M2 macrophage polarization within the tumor microenvironment, which in turn significantly promotes gastric cancer metastasis.

\section{IL-6 and IL-8 play important roles in GC-MSC-mediated macrophage polarization}

In this study, we further investigated the mechanism of M2-like polarization of macrophages prompted by GCMSCs, and the key factors were screened in the supernatant of GC-MSCs and GCN-MSCs. After analysis by Luminex assay, cytokines/chemokines including VEGF, MCP-1, IL-6, and IL-8 were detectable in the CM of GCMSCs and GCN-MSCs, whereas GC-MSCs produced a strikingly higher level of IL-6 and IL-8 secretion with significant differences from GCN-MSCs (Fig. 5a). Since both IL- 6 and IL- 8 are reported to play a paramount role in macrophage polarization ${ }^{21,22}$, we herein investigated the contribution of IL-6, IL-8, or IL-6 and IL-8 together to the promotion of macrophage phenotype switching by GC-MSCs. First, increased phosphorylation of JAK2 and STAT3 in macrophages polarized by GC-MSCs was observed to be partly abrogated by either IL-6- or IL-8specific neutralizing antibody treatment as shown in Fig. 5b. After treatment with neutralizing antibodies, both anti-IL-6 and anti-IL-8 antibodies significantly upregulated the proportion of $\mathrm{CD}^{+} 6^{+}$cells and reduced the proportion of CD204 ${ }^{+}$cells in GC-MSC-treated macrophage populations (Fig. 5c). In addition, we studied the role of JAK2/STAT3 signaling pathway in macrophage 


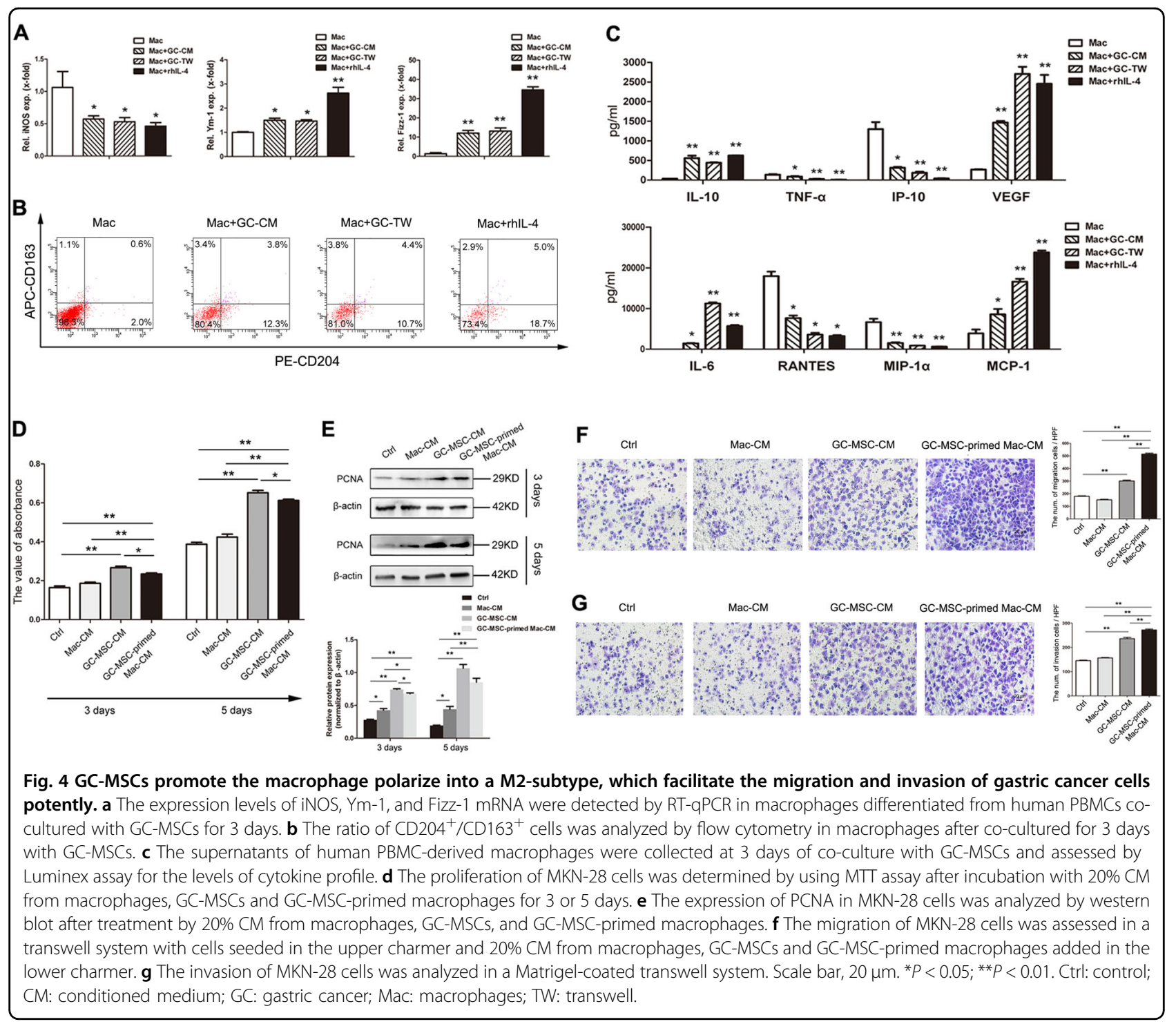

polarization by GC-MSCs, and the proportion of M2subtype macrophages was significantly downregulated by the application of selective JAK2 and STAT3 inhibitor (JSI-124, R\&D Systems, Minneapolis, MN, USA), as shown by the results of the flow cytometry (Fig. 5c). Thus, IL-6 or IL- 8 secreted by GC-MSCs partly mediated their regulating effect on macrophage polarization through the activation of the JAK2/STAT3 signaling pathway. Furthermore, we investigated the contribution of IL-6, IL-8 and the JAK2/STAT3 signaling pathway to the pro-tumor effect of GC-MSC-primed macrophages. The results showed that macrophages displayed an attenuated ability to promote the migration and invasion of gastric cancer cells after co-cultured with IL-6- or IL8-specific neutralizing antibody-pretreated GC-MSC$\mathrm{CM}$, or treated with JSI-124 before co-cultured with GCMSC-CM (Fig. 5d, e).

\section{GC-MSC-primed macrophages remarkably promote the} EMT process in gastric cancer cells

Previous studies have reported that macrophagesecreted cytokines can stimulate the process of epithelial mesenchymal transition (EMT), which is closely associated with cell migration ${ }^{23,24}$. Herein, we thoroughly studied whether promotion of gastric cancer metastasis by GC-MSC-primed macrophages is due to their promotion of the EMT process in gastric cancer cells. As shown in Fig. 6a, morphological changes in MKN-28 cells were first observed, which is one of the characteristics of EMT, after 5 days of treatment with either GC-MSC-CM or GCMSC-primed macrophage-CM. Moreover, the scattering degree of cancer cells increased remarkably, and the cell shape was considerably more elongated in MKN-28 cells treated by GC-MSC-primed macrophage-CM compared with GC-MSC-CM; morphological changes were not 


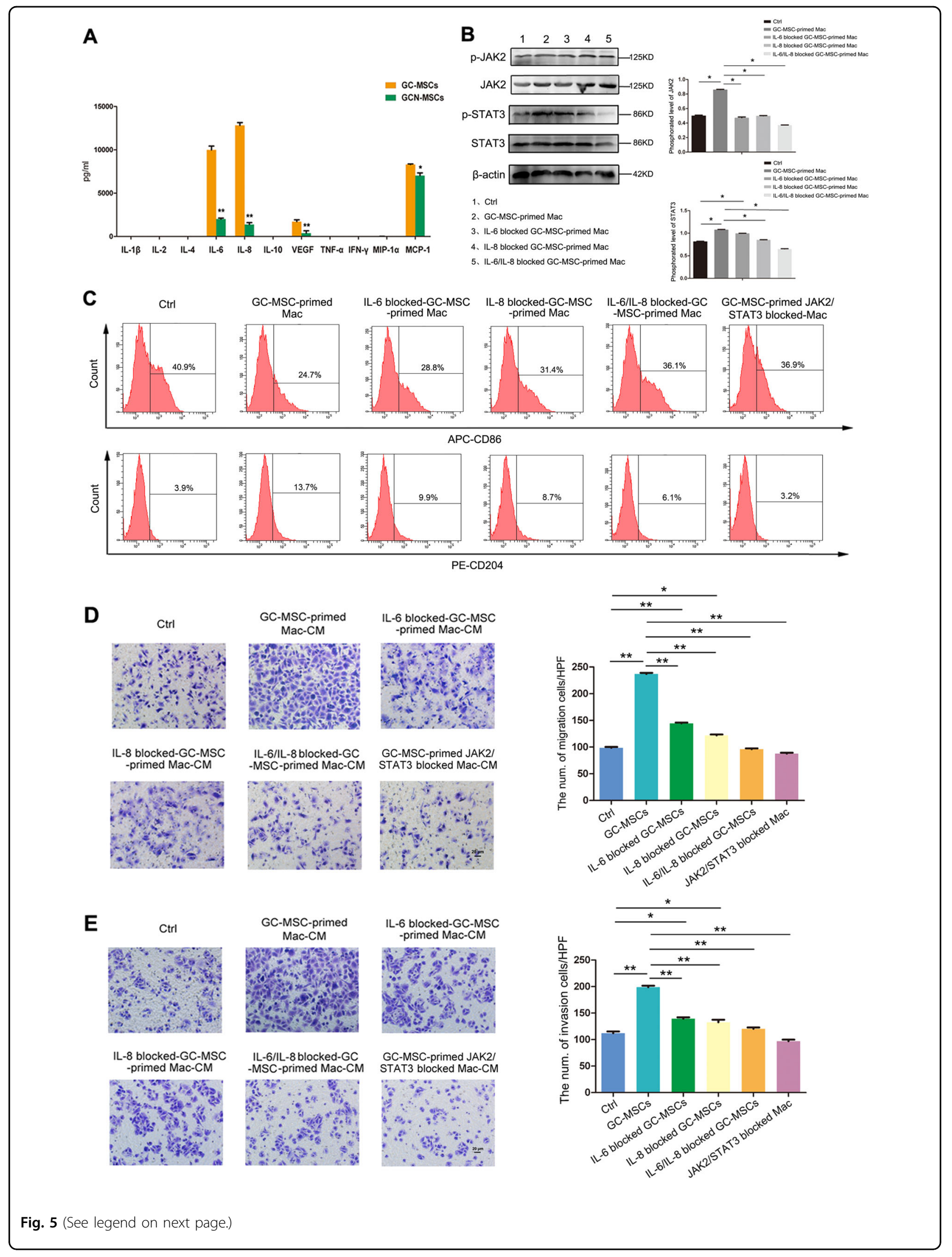


(see figure on previous page)

Fig. 5 Regulating-effect of GC-MSCs on the M2-polarization of macrophages was partly mediated by IL-6 and IL-8 via the JAK2/

STAT3 signaling pathway. a Cytokine profile analysis of GC-MSCs and GCN-MSCs by Luminex immunoassay. b Protein levels of JAK2, p-JAK2, STAT3, and p-STAT3 were detected by western blot in macrophages cultured in 20\% GC-MSC-CM and anti-IL6/IL-8 pretreated GC-MSC-CM. c The proportion of $\mathrm{CD} 6^{+}(\mathrm{M} 1)$ or $\mathrm{CD}_{204}^{+}(\mathrm{M} 2)$ cells was detected by flow cytometry in macrophages cultured in 20\% GC-MSC-CM and anti-IL6/IL-8 pretreated GCMSC-CM, or treated by selective JAK2/STAT3 inhibitor (JSI-124) before cultured in 20\% GC-MSC-CM. $\mathbf{d}$ The migration of MKN-28 cells was assessed in a transwell system with 20\% CM from GC-MSC-primed macrophages and IL-6/IL-8-blocked GC-MSC-primed macrophages, or treated by JSI-124 before cultured in 20\% GC-MSC-CM. e The invasion of MKN-28 cells was analyzed in a Matrigel-coated transwell system. Scale bar, $20 \mu \mathrm{m}$. ${ }^{*} P<0.05$;

${ }^{* *} P<0.01$. Ctrl: control; CM: conditioned medium; GC: gastric cancer; Mac: macrophages; TW: transwell.

obvious in MKN-28 cells treated with macrophage-CM, which were not pre-polarized by GC-MSCs (Fig. 6a). Colony formation assay revealed that $\mathrm{MKN}-28$ cells treated with GC-MSC-CM and GC-MSC-primed macrophage-CM both grew faster and formed larger colonies than the control and macrophage-CM-treated groups. In GC-MSC-primed macrophage-CM-treated gastric cancer cells, mesenchymal-like cell morphology was observed considerably with a largest colony phenotype than the other groups (Fig. 6b). In addition, the expression levels of EMT-related proteins including fibronectin, vimentin and snail2 increased most significantly in MKN-28 cells treated with GC-MSCprimed macrophage-CM compared with those in any other group; the level of E-cadherin notably decreased (Fig. 6c). In agreement with this, the RT-qPCR results indicated that at 5 days of co-culture, GC-MSC-primed macrophage-CM significantly upregulated the levels of fibronectin, vimentin and snail2 mRNA in MKN-28 cells, whereas the expression level of E-cadherin was reduced. These gene alterations were all more significant than those in GC-MSC-CM -treated gastric cancer cells (Fig. 6d). Thus, our data indicate that GC-MSC-primed macrophages could potently promote the progress of EMT in gastric cancer cells, which may be responsible for their striking promotion of gastric cancer cell migration.

\section{Discussion}

Herein, we demonstrated for the first time that macrophages play an essential role in the potent pro-tumor effect of GC-MSCs; in turn, GC-MSCs induce the polarization of macrophages into the M2 subtype through secreting IL-6 and IL-8 via the JAK2/ STAT3 signaling pathway. Subsequently, these GCMSC-primed macrophages display a prominent role in promoting the migration and invasion of gastric cancer cells via advancing the process of EMT. In total, our findings provide unique insight into the generation of pro-tumor M2-TAMs in gastric cancer. The generation of M2 macrophages 'educated' by GC-MSCs remarkably contributes to the re-shaping of the gastric cancer microenvironment and consequently affects the metastasis of gastric cancer.

Gastric cancer is regarded as a classic model of inflammation-related cancer, as well as one of the most common causes of cancer-related deaths worldwide ${ }^{25,26}$. Previously, we demonstrated that GC-MSCs play a dominant role in cancer angiogenesis and progression through the secretion of the cytokine IL- $8^{19}$. However, the interactions between GC-MSCs and immune cells within the tumor site may contribute to tumor progression and deserve thorough investigation. Zhu et al. ${ }^{27}$ reported that the reciprocal interaction between GC-MSCs and neutrophils presents a novel mechanism for the role of MSCs in remodeling the cancer niche, which in turn enhances the invasiveness of gastric cancer cells and angiogenesis. Herein, we propose that macrophages are an essential factor for the pro-tumor effect of GC-MSCs and focus on the generation and function of M2 macrophages. Ren et $\mathrm{al}^{28}$ reported that MSCs isolated from spontaneous lymphoma (L-MSCs) could enhance tumor growth more potently than BM-MSCs. They further identified that monocytes/macrophages, but not neutrophils, mediate the pro-tumor effect of L-MSCs, which is in support of our notion.

Macrophages, one of the major immune cells in the tumor stroma, have been demonstrated to interact with BM-MSCs in various types of tumor. Hashimoto et al. ${ }^{29}$ reported that BM-MSCs and PBMC-derived macrophages are recruited to tumor sites in neuroblastoma and activated to become cancer-associated fibroblasts and TAMs, respectively, which contributes to the formation of a favorable microenvironment for cancer progression. Yang et al. ${ }^{30}$ demonstrated that after activation by macrophages, BM-MSCs acquire a pro-inflammatory phenotype, which could promote gastric cancer cell proliferation and migration through NF- $\mathrm{B}$ activation. Another study by Wolfe et al. ${ }^{31}$ conveyed that the interactions between BM-MSCs and macrophages induce protumor properties such as enhanced migration and elevated IL-6 secretion, and macrophage-educated BMMSCs displayed enhanced invasion and mammosphere formation. 


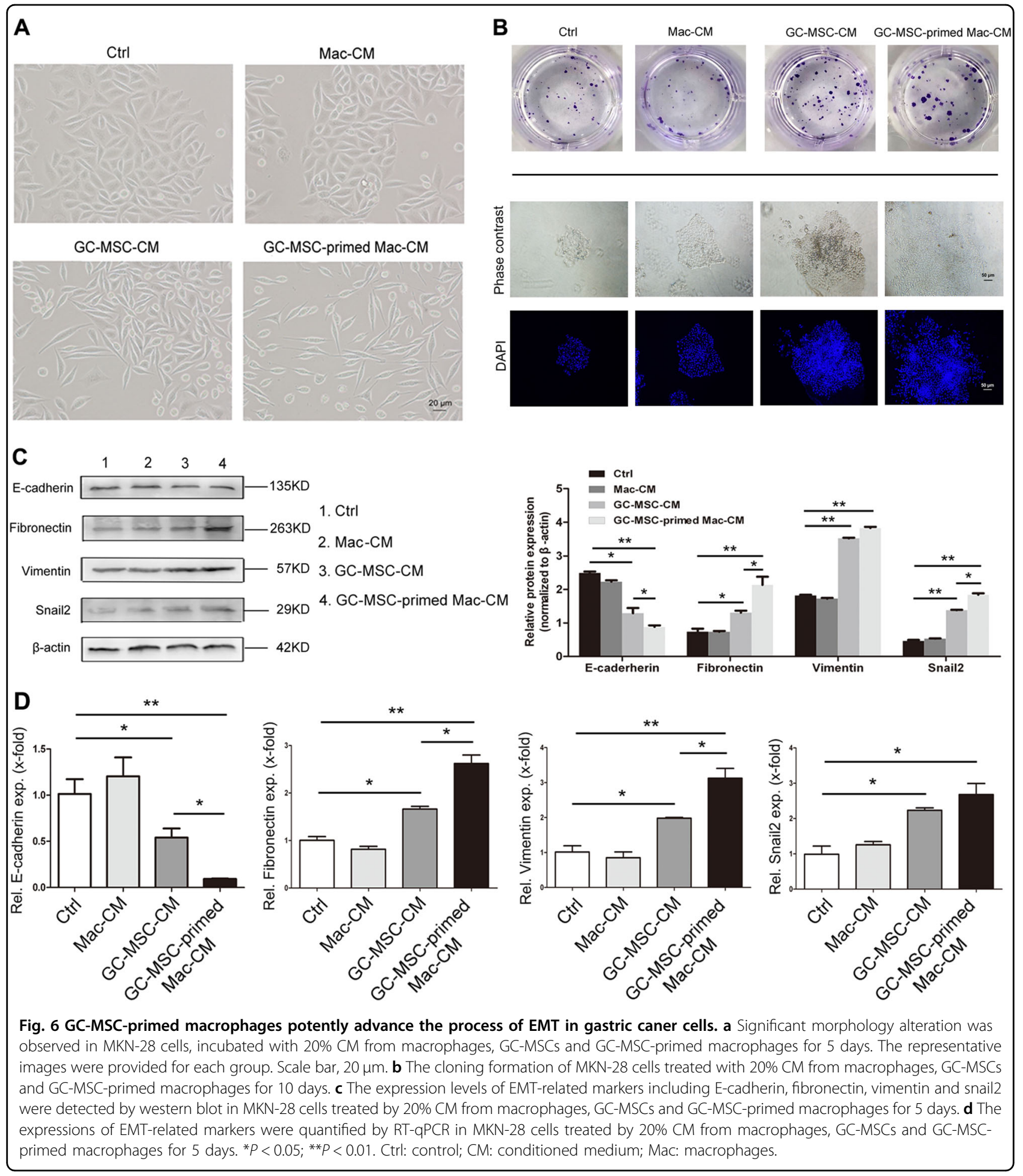

However, urgent clarification is needed on the regulation of the phenotypes and functions of macrophages by tumor tissue-derived MSCs and the underlying mechanism, which contributes to the formation of pro-tumor TAMs. Mathew et al..$^{32}$ identified a cell population named cancer-associated mesenchymal stem cells (CA-MSCs) in pancreatic cancer, whose ability to support tumor growth is associated with their promotion of alternative macrophage (M2) polarization. Yamada et al. ${ }^{33}$ also indicated that the angiogenic factor MFG-E8 drives melanoma growth through stimulating MSC-induced M2 polarization of TAMs. In the present study, we demonstrated that 
GC-MSCs are capable of converting the macrophages to an M2 phenotype partly mediated by IL- 6 and IL-8, and these educated macrophages can markedly promote gastric cancer metastasis by advancing EMT in gastric cancer cells.

First, high expression of M2 macrophage-related proteins was detected in gastric cancer tissues, where GCMSCs co-exist. Upon sorting cells from gastric cancer tissues by MACS technology, the TAMs displayed a significantly higher proportion of $\mathrm{CD} 204^{+}$cells and higher expression levels of genes indicating an M2-like immunophenotype compared to the macrophages derived from the adjacent non-cancerous tissues. Thereafter, the characterized differences in macrophages derived from human peripheral monocytes between gastric cancer patients and the healthy controls were also observed, suggesting that the phenotype of monocytes in peripheral blood was more inclined to be re-polarized to an M2 subtype in gastric cancer patients than in healthy controls.

Since the M2 subtype is the prominent phenotype of macrophages resident in GC-MSC co-existing gastric cancer tissues, we further confirmed the potential effect of GC-MSCs on macrophage polarization in an in vitro co-culture system. After co-culture for 3 days, we found that Ym-1 and Fizz-1, which are major M2 macrophagerelated genes, were both highly upregulated by GC-MSC treatment of macrophages. Moreover, a high proportion of $\mathrm{CD} 204^{+} / \mathrm{CD} 163^{+}$macrophages, as well as increasing levels of IL-6, IL-10, VEGF, and MCP-1 secretion by GCMSC-treated macrophages, synergistically revealed a potent role of GC-MSCs in polarizing macrophages into a pro-tumor (M2) phenotype. To convey the underlying mechanism, the cytokine/chemokine profile of GC-MSCs was screened, and IL- 6 and IL- 8 were highly secreted by GC-MSCs compared with GCN-MSCs. Thereafter, neutralizing antibodies were used, and IL- 6 and IL-8 were both confirmed as the key factors mediating the phenotype switch of macrophages by GC-MSCs. Furthermore, we also observed that GC-MSCs increased the activation of JAK2 and STAT3 in macrophages, and this effect could be partly abrogated in the presence of IL-6- or IL8-specific neutralizing antibody, indicating that GCMSCs promote the generation of M2 subtype TAMs by secreting IL-6 and IL-8 through regulating the JAK2/ STAT3 signaling pathway.

Furthermore, the role of GC-MSC-primed macrophages in gastric cancer progression was also further assessed in our study and GC-MSC-primed macrophages facilitated the proliferation of gastric cancer cells. However, the progrowth effect of GC-MSC-primed macrophages was less efficient than that of GC-MSCs, which has been previously identified to play a prominent promoting role in gastric cancer cell growth ${ }^{19}$. Hashimoto et al. $^{29}$ found that coculture with TAM-like macrophages did not enhance the proliferation but supported the invasive ability of tumor cells in neuroblastoma via CXCL2/CXCR2 signaling. Herein, we observed a remarkable ability of GC-MSCprimed macrophages to promote the migration and invasion of gastric cancer cells, which was even more potent than that of GC-MSCs, suggesting an important role for GC-MSC-educated TAMs in gastric cancer metastasis. These findings drew our attention and the underlying mechanism was further investigated. In addition, IL-6/IL-8specific neutralizing antibody and selective JAK2/STAT3 inhibitor treated GC-MSC-primed macrophages were also studied and their promoting effects on the migration and invasion abilities of gastric cancer cells were partly blocked, suggesting the key roles of IL- 6 and IL- 8 in macrophage polarization regulated by GC-MSCs.

EMT, one of the distinctive features of tumor metastasis, is a biological process characterized by cellular and molecular changes, including the loss of cell-to-cell adhesion, acquisition of a more spindle-like morphology and upregulation of the mesenchymal proteins ${ }^{34}$. In this study, a marked, slender morphological change was first observed in gastric cancer cells with GC-MSC-primed macrophage treatment compared with GC-MSCs. Liu et al. ${ }^{35}$ noted that M2-polarized TAMs can promote EMT in pancreatic cancer cells, partially by the TLR-4/IL10 signaling pathway. Yan et al. $^{36}$ reported that high infiltration of TAMs is related to poor prognosis and contributes to the phenomenon of EMT in gastric cancer. Herein, our investigation demonstrated that GC-MSCprimed macrophage-CM-treated gastric cancer cells formed larger colonies and considerably mesenchymallike cell morphology compared with the other groups. Moreover, the expression levels of vimentin, fibronectin and snail2, the three major mesenchymal genes, were all significantly upregulated in gastric cancer cells by GCMSC-primed macrophages compared with those in the other groups. In contrast, the expression level of E-cadherin, the key epithelial-related gene, was largely reduced. Therefore, our findings indicated that GC-MSC-educated macrophages could advance the process of EMT in gastric cancer cells, which might contribute to their prominent promotion of tumor metastasis.

\section{Conclusion}

In the present study, we highlight the unique ability of stromal GC-MSCs to induce the polarization of macrophages into a pro-tumor M2 subtype within the gastric cancer niche through the secretion of IL- 6 and IL- 8 via the JAK2/STAT3 signaling pathway. In turn, GC-MSCprimed macrophages remarkably promote gastric cancer metastasis by advancing the process of EMT in gastric cancer cells. Strategies that target the generation of protumor TAMs in tumor tissues may provide potential therapeutic targets for gastric cancer therapy. 


\section{Materials and methods \\ Cell culture}

GC-MSCs were isolated as described previously ${ }^{19}$. Tumor tissues were obtained from patients who underwent radical gastrectomy and the procedure was approved by the Ethics Committee of First People's Hospital of Lianyungang (Jiangsu, China). Briefly, fresh tumor tissues were cut into 1$\mathrm{mm}^{3}$-sized pieces after rinsing in antibiotics to avoid contamination and placed directly into culture dishes for $30 \mathrm{~min}$ to improve adhesion. Thereafter, the tissue explants were floated in a growing medium of L-DMEM (Gibco, Invitrogen Corporation, Carlsbad, CA, USA) containing $15 \%$ (v/v) fetal bovine serum (FBS; Gibco). When the spindle-like cells reached subconfluence, tissue pieces were removed and adherent cells were passaged into flasks for further expansion. The homogeneous cell population at 4-5 passages was used in this study.

Human gastric cancer cell lines BGC-823 and MKN-28 were gifts from the First Affiliated Hospital of Soochow University (Jiangsu, China) and cultured in 10\% (v/v) FBSRPMI 1640 (Gibco).

\section{Mouse xenograft models of gastric cancer}

All animal experiments were performed in accordance with the Guidelines and Regulations for Animal Experiments of First People's Hospital of Lianyungang. Threeto four-week-old female BALB/c nude (nu/nu) mice were purchased from the Nanjing Biomedical Research Institute of Nanjing University (Nanjing, China) and housed at the Animal Centre of Neurological Medical Institute in First People's Hospital of Lianyungang. To establish the xenograft models, a total of $5 \times 10^{6}$ gastric cancer cells (BGC-823 or MKN-28) in $200 \mu \mathrm{l}$ PBS were subcutaneously injected into the right flank near the axillary fossa of the mouse. To assess the pro-tumor effect of GCMSCs in vivo, $1 \times 10^{6}$ cells were subcutaneously coinjected in $200 \mu \mathrm{l} \mathrm{PBS}$. Animals were randomly divided into 5 mice per group and sacrificed at the end of the experiments for an observation period of 14 days. Tumor weights were recorded per mouse and tumor volumes were calculated by the modified formula of (length $x$ width $\left.^{2}\right) / 2$. Primary tumors were then collected for tissue section and homogenate preparation.

\section{Macrophage-depletion in the mouse xenograft model}

Clodronate [dichloromethylene bisphosphonate] liposome (lipo- $\mathrm{Cl}_{2} \mathrm{MBP}$ ) or liposomal vehicle (lipo-PBS) (Nicovan Rooijen, Amsterdam, Netherlands) was given to the mice transplanted with subcutaneous tumors every 4 days by tail vein injection at a dose of $100 \mu \mathrm{l} / 10 \mathrm{~g}$ mouse.

\section{Morphological analysis and immunohistochemistry}

Tumor tissues collected from each group of mice were fixed in $4.0 \%$ paraformaldehyde, embedded in paraffin and cut into 4- $\mu \mathrm{m}$ sections. $\mathrm{H} \& \mathrm{E}$ staining was performed and used to measure the tumor burden. On the other hand, tumor sections were incubated with 3.0\% hydrogen peroxide to inactivate endogenous peroxidase. After antigen retrieval, the sections were blocked in $5.0 \%$ bovine serum albumin and incubated with the following primary antibodies at $4{ }^{\circ} \mathrm{C}$ overnight: rabbit anti-mouse $\mathrm{F} 4 / 80$ (ab100790; Abcam, Cambridge, MA, USA), CD31 (ab28364, Abcam) and Ki67 (ab16667, Abcam). After washing with PBS, further incubation with a biotin-conjugated secondary antibody was performed, followed by visualization with $3,3^{\prime}$ diaminobenzidine (DAB) and counterstaining with hematoxylin for examination on an inverted microscope (IX71, Olympus, Tokyo, Japan).

\section{RNA isolation and RT-qPCR}

Total RNA was extracted from tumor homogenate or cell lines using TRIzol reagent (Life Technologies, Invitrogen, Carlsbad, CA, USA) and cDNA synthesis was processed by a reverse transcription kit according to the manufacturer's instructions (Roche Diagnostics, Indianapolis, IN, USA). qPCR was performed in a reaction mixture containing $10 \mu \mathrm{l}$ $2 \times$ SYBR Taq (Tli Plus) (Takara), $0.4 \mu \mathrm{l} 10 \mu \mathrm{M}$ of each primer, $0.4 \mu \mathrm{l} 50 \times$ ROX Reference Dye II (Takara), and $2 \mu \mathrm{l}$ cDNA on an Applied Biosystems 7500 Cycler (Applied Biosystems). Primers were designed using the Primer Software produced by Invitrogen Company (Table 1). The relative expression level was determined using the $2^{-\Delta \Delta C t}$ analysis method, where $\beta$-actin was used as the internal standard. All reactions were run in triplicate.

\section{Western blot analysis}

Tumor homogenate or cell lysate was prepared with RIPA buffer supplemented with complete protease inhibitors. Equal amounts of proteins were separated in $8 \%$ or $12 \%$ SDS-PAGE and immunoblotted with the following primary antibodies: anti-arginase-1 (MABS388, MerckMillipore, Billerica, MA, USA), anti-CCR-2 (ab125686, Abcam), anti-PCNA (NA03, Merck-Millipore), anti-pJAK2 (4406T, Cell Signaling Technology, Beverly, MA, USA), anti-JAK2 (3230T, Cell Signaling Technology), anti-p-STAT3 (9145T, Cell Signaling Technology), antiSTAT3 (4904T, Cell Signaling Technology), antifibronectin (ab32419, Abcam), anti-vimentin (\#5741P, Cell Signaling Technology), anti-snail2 (\#3879P, Cell Signaling Technology), and anti- $\beta$-actin (ab8226, Abcam), followed by the secondary HRP-conjugated antibodies.

\section{Immunofluorescence histochemistry}

Tumor tissues collected from gastric cancer patients were fixed in $4.0 \%$ paraformaldehyde, embedded in paraffin and cut into 4- $\mu \mathrm{m}$ sections. Sections were incubated with $3.0 \%$ hydrogen peroxide to inactivate endogenous peroxidase and then blocked in $5.0 \%$ bovine serum 
Table 1 Primer sequences of target genes.

\begin{tabular}{|c|c|c|c|}
\hline Genes & Primer sequence $\left(5^{\prime}-3^{\prime}\right)$ & $\begin{array}{l}\text { Product } \\
\text { Size (bp) }\end{array}$ & $\begin{array}{l}\text { Annealing } \\
\text { Temperature }\left({ }^{\circ} \mathrm{C}\right)\end{array}$ \\
\hline \multirow[t]{2}{*}{$\mathrm{H}$-iNOS } & For: TGCCACCTCCAGTCCAGTGA & 129 & 62 \\
\hline & Rev: GCATCCAGCTTGACCAGAGA & & \\
\hline \multirow[t]{2}{*}{$\mathrm{H}-\mathrm{Ym}-1$} & For: CCAGTGCTGCTCTGCATACA & 189 & 62 \\
\hline & Rev: ATGCCGTAGAGCGTCACATC & & \\
\hline \multirow[t]{2}{*}{ H-Fizz-1 } & For: CGTCCTCTTGCCTCCTTCTC & 227 & 62 \\
\hline & Rev: ACAAGCACAGCCAGTGACAG & & \\
\hline \multirow[t]{2}{*}{ H-CD163 } & For: ACTCTGGATCTGCTGACTTC & 219 & 60 \\
\hline & Rev: CTTCCATGCTCCAGCCATTA & & \\
\hline \multirow[t]{2}{*}{$\mathrm{H}-\mathrm{CD} 204$} & For: AATAGTGGCAGCTCAACTCC & 201 & 61 \\
\hline & Rev: ATGAGGTTGGCTTCCATGTC & & \\
\hline \multirow[t]{2}{*}{$\mathrm{H}-\mathrm{CD} 206$} & For: ATTAGGTGGAGAGGCAGTTG & 259 & 62 \\
\hline & Rev: GTGATTCGGCATCCTGGTTG & & \\
\hline \multirow[t]{2}{*}{ H-IL-10 } & For: GATCTCCGAGATGCCTTCAG & 259 & 61 \\
\hline & Rev: ATCGATGACAGCGCCGTAGC & & \\
\hline \multirow[t]{2}{*}{$\mathrm{H}-\mathrm{CCL}-22$} & For: GGCGCTTCAAGCAACTGAGG & 260 & 65 \\
\hline & Rev: CAAGGCCACGGTCATCAGAG & & \\
\hline \multirow[t]{2}{*}{$\mathrm{H}-\mathrm{IL}-23$} & For: CCAGAGACGCGCTGAACAGA & 606 & 64 \\
\hline & Rev: TTGAAGCGGAGAAGGAGACG & & \\
\hline \multirow[t]{2}{*}{$\mathrm{H}$-E-cadherin } & For: GCCGAGAGCTACACGTTCAC & 355 & 60 \\
\hline & Rev: GCTITCTGAGGCCAGGAGAG & & \\
\hline \multirow[t]{2}{*}{ H-fibronectin } & For: GCGGACCTACCTAGGCAATG & 269 & 62 \\
\hline & Rev: TCCTCCAGGTGTCACCAATC & & \\
\hline \multirow[t]{2}{*}{$\mathrm{H}$-vimentin } & For: CGCCAACTACATCGACAAGG & 306 & 62 \\
\hline & Rev: GCCAGAGACGCATTGTCAAC & & \\
\hline \multirow[t]{2}{*}{ H-snail2 } & For: ACTACAGCGAACTGGACACA & 743 & 60 \\
\hline & Rev: ACAGCAGCCAGATTCCTCAT & & \\
\hline \multirow[t]{2}{*}{$H-\beta$-actin } & For: TGGACTTCGAGCAAGAGATG & 207 & 60 \\
\hline & Rev: GGATGTCCACGTCACACTTC & & \\
\hline \multirow[t]{2}{*}{ M-iNOS } & For: ACTGCAAGAGAACGGAGAAC & 347 & 59 \\
\hline & Rev: GCCTTGTGGTGAAGAGTGTC & & \\
\hline \multirow[t]{2}{*}{$M-Y m-1$} & For: CCAGTGCTGCTCTGCATACA & 189 & 62 \\
\hline & Rev: ATGCCGTAGAGCGTCACATC & & \\
\hline \multirow[t]{2}{*}{$M-\beta$-actin } & For: ACCAGTTCGCCATGGATGAC & 299 & 60 \\
\hline & Rev: CACACGCAGCTCATTGTAGA & & \\
\hline
\end{tabular}

albumin after antigen retrieval. To observe the relationship between GC-MSCs and TAM location within tumor, the sections were incubated at $4{ }^{\circ} \mathrm{C}$ overnight with primary antibodies against $\alpha-S M A$ (NBP2-33006, Novus Biologicals, Briarwood Avenue, CO, USA) and CD204 (bs136214, absin, Shanghai, China). Subsequently, incubation with Alexa Fluor 488-labelled goat anti-mouse IgG $(\mathrm{H}+\mathrm{L})$ and Alexa Fluor 555-labelled donkey anti-rabbit IgG $(\mathrm{H}+\mathrm{L})$ (Beyotime, Shanghai, China) was performed for $1 \mathrm{~h}$ at $37^{\circ} \mathrm{C}$ in the dark. Nuclei were counterstained 
with DAPI (Beyotime). Fluorescent images were acquired by a confocal laser-scanning microscope (Ti2-E-A1, Nikon, Tokyo, Japan).

\section{Selection of human gastric cancer-derived TAMs}

Gastric cancer and adjacent non-cancerous tissues were obtained from patients who underwent radical gastrectomy. After rinsing in antibiotics, the primary tumor tissues were dissociated into single-cell suspensions by a human tumor dissociation kit (130-095-929, Miltenyi Biotech, Cologne, Germany) according to the manufacturer's instructions on a gentleMACS ${ }^{\mathrm{TM}}$ Octo Dissociator with Heaters (Miltenyi Biotech). Thereafter, TAMs were obtained by performing positive selection with magnetically labeled human CD14 beads (Miltenyi Biotech) according to the manufacturer's instructions.

\section{Preparation of human peripheral monocyte-derived macrophages}

First, peripheral blood mononuclear cells (PBMCs) were isolated by Ficoll-Hypaque (1.077 g) (LTS1077, tbdscience, Tianjin, China). Then, CD14 ${ }^{+}$cells were isolated from the PBMCs by performing CD14 bead positive selection (Miltenyi Biotech) according to the manufacturer's instructions. After confirming the purity of $\mathrm{CD}_{1} 4^{+}$ monocytes by flow cytometry, the cells were cultured in 6well plates at $5 \times 10^{5}$ cells/ml in $10 \%$ FBS-RPMI 1640 and differentiated into macrophages by incubation with $50 \mathrm{ng} / \mathrm{ml}$ phorbol 12-myristate 13-acetate (PMA) for $24 \mathrm{~h}$.

\section{Flow cytometry}

In this study, flow cytometric analysis was used to detect the immunophenotype of macrophages in gastric cancer tissues, peripheral blood or a co-culture system with GCMSCs. Briefly, a single-cell suspension of macrophages was first incubated with PE-CD204 (FAB2708P, R\&D Systems), APC-CD163 (ab134416, Abcam) or APC-CD86 (560956, BD Pharmingen, San Jose, CA, USA) for $30 \mathrm{~min}$ at $4{ }^{\circ} \mathrm{C}$ in the dark. After washing, the labeled cells were resuspended in PBS and analyzed on a FACSCanto II flow cytometer (BD Biosciences, Sparks, MD, USA). As negative controls, isotype-matched antibodies with the corresponding fluorescent labels were used.

\section{Luminex immunoassay}

In a co-culture system with GC-MSCs, the cytokine profile of macrophages was analyzed by Luminex assay. After 3 days of co-culture, the supernatant of cell cultivation media was collected so that the concentration and culture time were coincident to make the data comparable. Then, the levels of IL-6, IL-10, tumor necrosis factor- $\alpha$ (TNF- $\alpha$ ), IFN- $\gamma$-induced protein 10 (IP-10), C-C motif chemokine ligand 5 (CCL5/RANTES), vascular endothelial growth factor (VEGF), macrophage inflammatory protein-
$1 \alpha(\mathrm{MIP}-1 \alpha)$ and monocyte chemoattractant protein-1 (MCP-1) were quantified simultaneously using a human cytokine/chemokine kit (MPXHCYTO-60K, Merck-Millipore) on a multiplexed bead array immunoassay platform (Bio-Plex 200, Bio-Rad Laboratories, Hercules, CA, USA). All reactions were run in triplicate. The differences in cytokine profile between GC-MSCs and MSCs from adjacent non-cancerous gastric cancer tissues (GCNMSCs) were also analyzed by Luminex immunoassay.

\section{MTT assay}

In 96-well plates, MKN-28 cells $\left(2 \times 10^{4}\right.$ cells $\left./ \mathrm{ml}\right)$ were incubated with $20 \%$ CM from macrophages, GC-MSCs or GC-MSC-primed macrophages, respectively. At 3 or 5 days, MTT solution $(5 \mathrm{mg} / \mathrm{ml})$ was added into each well and incubated with the cells at $37^{\circ} \mathrm{C}$ for $4 \mathrm{~h}$. Then, the solution was discarded and $150 \mu \mathrm{L}$ DMSO was added into the remaining cells for dissolving formazan crystals. The optical density (OD) at $490 \mathrm{~nm}$ was measured by a microplate reader (Bio-Rad Laboratories).

\section{Cell migration and invasion analysis}

To examine the effects of GC-MSC-primed macrophages on gastric cancer cell migration and invasion, cell assays were performed in a 24-well transwell chamber. For the migration assay, MKN-28 cells were seeded in serum-free RPMI-1640 at a density of $2 \times 10^{5}$ cells $/ \mathrm{ml}$ in the upper chamber of transwell inserts. For cell invasion assays, cells at $3 \times 10^{5}$ cells $/ \mathrm{ml}$ were plated in the upper compartment with Matrigel-coated membranes (Corning Life Sciences, Bedford, MA, USA). Twenty percent CM from macrophages, GC-MSCs or GC-MSC-primed macrophages was added into the lower chamber. After incubation at $37^{\circ} \mathrm{C}$ for $24 \mathrm{~h}$, the inserts were discarded and the upper side of the filter was swabbed to remove the nonmigratory cells. After fixation in $4 \%$ paraformaldehyde, the filters were stained with $0.5 \%$ crystal violet for $20 \mathrm{~min}$. The stained migrated or invaded cells were visualized and photographed by an inverted microscope (IX71), and 8 randomly non-overlapping high-power fields (HPFs, $\times 200$ ) were selected for counting the migrated cells.

\section{Neutralization assay by IL-6/IL-8 blockade}

To evaluate the effect of IL-6 or IL- 8 on M2-like macrophage polarization prompted by GC-MSCs, a neutralization assay was performed in our study. Briefly, macrophages were treated with 20\% GC-MSC-CM alone or together with a human IL-6 neutralizing antibody (554541, BD Pharmingen) at a final concentration of $2 \mathrm{ng} / \mathrm{ml}$, or with a human IL-8 neutralizing antibody (554726, BD Pharmingen) at a final concentration of $150 \mu \mathrm{g} / \mathrm{ml}$, or with a mixture of IL- 6 and IL-8 neutralizing antibodies. Mixtures of GC-MSC-CM and neutralizing antibodies were incubated at $4{ }^{\circ} \mathrm{C}$ overnight before being 
added to macrophages. The immunophenotype and macrophage polarization-related signaling pathway were assessed after neutralizing antibody treatment.

\section{Colony formation assay}

The colony forming ability of MKN-28 cells was determined in duplicate at 200 cells/well in a 24-well plate. The tumor cells were incubated in $20 \% \mathrm{CM}$ from macrophages, GC-MSCs or GC-MSC-primed macrophages, respectively, at $37^{\circ} \mathrm{C}$ in a humidified incubator. After 10 days, the adherent cells were washed with PBS, fixed with $4 \%$ paraformaldehyde and stained with $0.5 \%$ Crystal violet or DAPI.

\section{Statistical analysis}

The results are expressed as the mean \pm SEM. Data were analyzed with GraphPad Prism software 6.0 (GraphPad Software, La Jolla, CA, USA). Statistical analysis was performed by non-parametric Mann-Whitney $U$ test with SPSS 16.0 statistical software. $P<0.05$ was considered statistically significant.

\section{Acknowledgements}

This study was supported by the National Natural Science Foundation of China (Grant No. 81402280, 81672416, 81802347), the Natural Science Foundation of Jiangsu Province (Grant No.BK20161296), the Jiangsu Postdoctoral Research Foundation (Grant No.1501079 A), the Foundation from Jiangsu Key Laboratory of Medical Science and Laboratory Medicine (JSKLM-2014-005), the Doctoral Foundation from the First People's Hospital of Lianyungang (Grant number: BS1503).

\section{Author details}

'Center of Research Laboratory, The First People's Hospital of Lianyungang, Lianyungang 222001, China. ${ }^{2}$ Department of Pathology, Xuzhou Medical University, Xuzhou 221004, China. ${ }^{3}$ Jiangsu Key Laboratory of Medical Science and Laboratory Medicine, Jiangsu University, Zhenjiang 212013, China. ${ }^{4}$ Department of Oncology, The First People's Hospital of Lianyungang, Lianyungang 222001, China. ${ }^{5}$ Department of Gastrointestinal Surgery, The First People's Hospital of Lianyungang, Lianyungang 222001, China. ${ }^{6}$ Department of Clinical Laboratory Diagnostics, Kangda College of Nanjing Medical University, Lianyungang 222000, China

\section{Conflict of interest}

The authors declare that they have no conflict of interest.

\section{Publisher's note}

Springer Nature remains neutral with regard to jurisdictional claims in published maps and institutional affiliations.

Supplementary Information accompanies this paper at (https://doi.org/ 10.1038/s41419-019-2131-y).

Received: 19 August 2019 Revised: 3 November 2019 Accepted: 5 November 2019

Published online: 04 December 2019

\footnotetext{
References

1. Hong, J. K. \& Sang, C. O. Novel systemic therapies for advanced gastric cancer. J. Gastric Cancer 18, 1-19 (2018).
}

2. Francesco, F. et al. Can the addition of radiotherapy postoperatively increase clinical outcome of patients with gastric cancer? A systematic review of the literature and meta-analysis. Oncotarget 9, 10734-10744 (2018).

3. Korivi, B. R. et al. Intestinal and diffuse gastric cancer: a retrospective study comparing primary sites. Clin. Imaging 56, 33-40 (2019).

4. Mingjing, S. et al. Increased infiltration of macrophages to radioresistant lung cancer cells contributes to the development of the additional resistance of tumor cells to the cytotoxic effects of NK cells. Int. J. Oncol. 53, 317-328 (2018)

5. Pathria, P., Louis, T. L. \& Varner, J. A. Targeting tumor-associated macrophages in cancer. Trends Immunol. 40, 310-327 (2019).

6. Chongyu, S., Xiaolong, F., Wei, D., Peiwu, Y. \& Yongliang, Z. High density of CD68+ tumor-associated macrophages predicts a poor prognosis in gastric cancer mediated by IL-6 expression. Oncol. Lett. 15, 6217-6224 (2018).

7. Zhangting, Y. et al. Imatinib prevents lung cancer metastasis by inhibiting M2like polarization of macrophages. Pharmacol. Res. 133, 121-131 (2018).

8. Lutian, Y. et al. Interleukin-27 inhibits malignant behaviors of pancreatic cancer cells by targeting M2 polarized tumor associated macrophages. Cytokine $\mathbf{8 9}$, 194-200 (2017).

9. Pedraza-Brindis, E. J. et al. Culture supernatants of cervical cancer cells induce an M2 phenotypic profile in THP-1 macrophages. Cell Immunol. 310, 42-52 (2016).

10. Guoliang, Z. et al. A novel role of breast cancer-derived hyaluronan on inducement of M2-like tumor-associated macrophages formation. Oncoimmunology 5, e1172154 (2016).

11. Takahisa, Y. et al. Tumor-associated macrophages of the M2 phenotype contribute to progression in gastric cancer with peritoneal dissemination. Gastric Cancer 19, 1052-1065 (2016).

12. Alice, E. D., Edward, W. R. \& Douglas, T. F. Stromal cells in the tumor microenvironment. Adv. Exp. Med. Biol. 1060, 99-114 (2018).

13. Hanxiang, Z. et al. Crosstalk between stromal cells and cancer cells in pancreatic cancer: New insights into stromal biology. Cancer Lett. 392, 83-93 (2017).

14. Chen, Z. et al. The distinct roles of mesenchymal stem cells in the initial and progressive stage of hepatocarcinoma. Cell Death Dis. 9, 345 (2018).

15. Xiaochao, Z. et al. Human colorectal cancer-derived mesenchymal stem cells promote colorectal cancer progression through IL-6/JAK2/STAT3 signaling. Cell Death Dis. 9, 25 (2018).

16. Mei, W. et al. Gastric cancer tissue-derived mesenchymal stem cells impact peripheral blood mononuclear cells via disruption of Treg/Th17 balance to promote gastric cancer progression. Exp. Cell Res. 361, 19-29 (2017).

17. Galland, S. et al. Tumor-derived mesenchymal stem cells use distinct mechanisms to block the activity of natural killer cell subsets. Cell Rep. 20, 2891-2905 (2017).

18. Wei, L. et al. Macrophages are involved in the protective role of human umbilical cord-derived stromal cells in renal ischemia-reperfusion injury. Stem Cell Res. 10, 405-416 (2013).

19. Wei, L. et al. Gastric cancer-derived mesenchymal stem cells prompt gastric cancer progression through secretion of interleukin-8. J. Exp. Clin. Cancer Res. 34, 52 (2015).

20. Nico, V. R. \& Annemarie, S. Liposome mediated depletion of macrophages: mechanism of action, preparation of liposomes and applications. J. Immunol. Methods 174, 83-93 (1994).

21. Bin, J., Shujuan, Z., Songshu, X., Min, X. MiR-217 Inhibits M2-like macrophage polarization by suppressing secretion of interleukin-6 in ovarian cancer. Inflammation: https://doi.org/10.1007/s10753-019-01004-2 (2019).

22. Yingxia, N. et al. Genistein inhibits stemness of SKOV3 cells induced by macrophages co-cultured with ovarian cancer stem-like cells through IL-8/ STAT3 axis. J. Exp. Clin. Cancer Res. 38, 19 (2019).

23. Jia, Z. et al. High infiltration of tumor-associated macrophages influences poor prognosis in human gastric cancer patients, associates with the phenomenon of EMT. Medicine 95, e2636 (2016).

24. Jianhui, C. et al. Tumor-associated macrophages derived TGF-ß-induced epithelial to mesenchymal transition in colorectal cancer cells through Smad2,34/Snail signaling pathway. Cancer Res. Treat. 51, 252-266 (2019).

25. Kazım, S., Murat, B. Ö., Selahattin, V. \& Mesut, T. The role of inflammation in gastric cancer. Adv. Exp. Med. Biol. 816, 235-257 (2014).

26. Kanae, E., Osamu, H., Yusuke, M. \& Masanobu, O. Inflammation in gastric cancer: interplay of the COX-2 /prostaglandin E2 and Toll-like receptor/MyD88 pathways. Cancer Sci. 107, 391-397 (2016). 
27. Qiongfang, Z. et al. The IL-6-STAT3 axis mediates a reciprocal crosstalk between cancer-derived mesenchymal stem cells and neutrophils to synergistically prompt gastric cancer progression. Cell Death Dis. 5, e1295 (2014).

28. Guangwen, R. et al. CCR2-dependent recruitment of macrophages by tumor educated mesenchymal stromal cells promotes tumor development and is mimicked by TNF-a. Cell Stem Cell 11, 812-824 (2012).

29. Okito, H. et al. Collaboration of cancer-associated fibroblasts and tumourassociated macrophages for neuroblastoma development. J. Pathol. 240 211-223 (2016)

30. Tingting, $Y$. et al. Activation of mesenchymal stem cells by macrophages prompts human gastric cancer growth through NF-kB pathway. PLoS ONE $\mathbf{9}$ e97569 (2014)

31. Adam, R. W. et al. Mesenchymal stem cells and macrophages interact through IL-6 to promote inflammatory breast cancer in pre-clinical models. Oncotarget 7, 82482-82492 (2016)
32. Esha, M. et al. Mesenchymal stem cells promote pancreatic tumor growth by inducing alternative polarization of macrophages. Neoplasia 18, 142-151 (2016).

33. Kazuya, Y. et al. MFG-E8 drives melanoma growth by stimulating mesenchymal stromal cell-induced angiogenesis and $M 2$ polarization of tumorassociated macrophages. Cancer Res. 76, 4283-4292 (2016).

34. Bethany, N. S. \& Neil, A. B. Role of EMT in metastasis and therapy resistance. J. Clin. Med. 5, 2 (2016).

35. Chaoying, L. et al. M2-polarized tumor-associated macrophages promoted epithelial-mesenchymal transition in pancreatic cancer cells, partially through TLR4/IL-10 signaling pathway. Lab. Invest. 93, 844-854 (2013).

36. Yan, Y. et al. High tumor-associated macrophages infiltration is associated with poor prognosis and may contribute to the phenomenon of epithelialmesenchymal transition in gastric cancer. Onco. Targets Ther. 9, 3975-3983 (2016). 